\title{
SOME CONDITIONS UNDER WHICH A HOMOGENEOUS CONTINUUM IS A SIMPLE CLOSED CURVE ${ }^{1}$
}

\section{E. BURGESS}

In a recent paper [3], the author added a note in proof stating that two of the results could be strengthened by using the fact that a nondegenerate continuous curve is a simple closed curve if it is nearly homogeneous and is not a triod. It is the main purpose of this note to present a proof of this theorem and to state stronger forms of two results in [3]. Also, a theorem is presented that is related to a question raised by Knaster and Kuratowski [6]. This question as to whether every nondegenerate homogeneous bounded plane continuum is a simple closed curve has been settled negatively with examples by Bing [1] and Bing and Jones [2]. Additional conditions under which there is an affirmative answer have been given in some of the references cited in [3]. It apparently has not been noticed previously that there is an affirmative answer for nondegenerate bounded continua that are homogeneously embedded in the plane. This result (Theorem 3) follows directly from a characterization of homogeneous decomposable bounded plane continua given by Jones [5] and the nonaccessibility of certain points of indecomposable plane continua [7].

Definitions. A continuous curve is a compact metric space that is connected and locally connected. A triod is a continuum which is separated into three mutually separated sets by one of its subcontinua. A continuum $M$ is: (i) homogeneous if for any two points $x$ and $y$ of $M$ there is a homeomorphism of $M$ onto itself that carries $x$ into $y$; (ii) nearly homogeneous if for any point $x$ of $M$ and any subset $D$ of $M$, open relative to $M$, there is a homeomorphism of $M$ onto itself that carries $x$ into a point of $D$; (iii) 2-homogeneous if for any two points $x_{1}$ and $x_{2}$ of $M$ and any two points $y_{1}$ and $y_{2}$ of $M$ there is a homeomorphism of $M$ onto itself that carries $x_{1}+x_{2}$ onto $y_{1}+y_{2}$; (iv) nearly 2-homogeneous if for any two points $x_{1}$ and $x_{2}$ of $M$ and any two subsets $D_{1}$ and $D_{2}$ of $M$ that are open relative to $M$ there exist two points $y_{1}$ and $y_{2}$ in $D_{1}$ and $D_{2}$, respectively, and a homeomorphism of $M$ onto itself that carries $x_{1}+x_{2}$ onto $y_{1}+y_{2}$; (v) homogeneously embedded in a space $S$ if for any two points $x$ and $y$ of $M$ there is a homeomorphism of $S$ onto itself that carries $x$ into $y$ and $M$ onto itself.

Presented to the Society, November 22, 1958; received by the editors October 3 , 1958.

1 This work was supported by the National Science Foundation under G-2574. 
THEOREM 1. If the nondegenerate continuous curve $M$ is nearly homogeneous and is not a triod, then $M$ is a simple closed curve.

Proof. There exist a closed subset $K$ of $M$ and two points $p_{1}$ and $p_{2}$ of $M$ such that $K$ is irreducible with respect to the property of separating $p_{1}$ from $p_{2}$ in $M$ and $M-K$ is the sum of two mutually separated sets $M_{1}$ and $M_{2}$ that contain $p_{1}$ and $p_{2}$, respectively. Let $G_{1}, G_{2}, G_{3}, \cdots$ be a sequence of finite collections of connected open subsets of $M$ such that for each $i$, (1) $G_{i}$ covers $K$, (2) each element of $G_{i}$ intersects $K$ and has a diameter less than $1 / i$, and (3) each element of $G_{i+1}$ is a subset of some element of $G_{i}$. There is a finite collection $T_{1}$ of arcs such that (1) $T_{1}^{*}$ is connected, ${ }^{2}$ (2) each arc of $T_{1}$ intersects an element of $G_{1}$, and (3) each element of $G_{1}$ intersects an arc of $T_{1}$. Now define a sequence $T_{1}, T_{2}, T_{3}, \ldots$ of finite collections of arcs such that for each $i(i>1)$, (1) each arc of $T_{i}$ is a subset of an element of $G_{i-1}$ and intersects an arc of $T_{i-1}$, (2) each arc of $T_{i}$ intersects an element of $G_{i}$, and (3) each element of $G_{i}$ intersects an arc of $T_{i}$. Let $K^{\prime}$ denote the continuum $K+T_{1}^{*}+T_{2}^{*}+\cdots$.

Suppose that $M$ is not a simple closed curve. That $K^{\prime}$ contains neither $M_{1}$ nor $M_{2}$ follows from the fact that a compact metric continuum is a simple closed curve provided it is nearly homogeneous and some arc in it contains a set that is open relative to that continuum. Hence $M-K^{\prime}$ is the sum of two mutually separated sets $M_{1}^{\prime}$ and $M_{2}^{\prime}$, where $M_{i}^{\prime}=M_{i}-K^{\prime}(i=1,2)$. Since $K^{\prime}-K$ is nowhere dense in $M$ and each point of $K$ is a limit point of both $M_{1}$ and $M_{2}$, it follows that each point of $K$ is a limit point of both $M_{1}^{\prime}$ and $M_{2}^{\prime} .^{3}$

Now from the near-homogeneity of $M$, it follows that there is a homeomorphism $f$ of $M$ onto itself that carries some point $x$ of $K$ into $M_{1}^{\prime}$. Then the point $f(x)$ is a limit point of both $f\left(M_{1}^{\prime}\right)$ and $f\left(M_{2}^{\prime}\right)$, so that $M_{1}^{\prime}$ intersects both $f\left(M_{1}^{\prime}\right)$ and $f\left(M_{2}^{\prime}\right)$. There exists an arc $H$ in $M$ such that $H+K^{\prime}+f\left(K^{\prime}\right)$ is a continuum that is nowhere dense in $M$. Let $N=H+K^{\prime}+f\left({ }^{\prime} K\right)$. Then $M-N$ is the sum of the three mutually separated sets $M_{2}^{\prime}-N, M_{1}^{\prime} \cdot f\left(M_{1}^{\prime}\right)-N$, and $M_{1}^{\prime} \cdot f\left(M_{2}^{\prime}\right)-N$, and this is contrary to the hypothesis that $M$ is not a triod. Hence $M$ is a simple closed curve.

Question. If the continuous curve $M$ has no local separating point and $p_{1}$ and $p_{2}$ are two points of $M$, then does there exist a subcontinuum $K$ of $M$ such that (1) $M-K$ is the sum of two mutually

2 If $L$ is a collection of point sets, then $L^{*}$ denotes the set which is the sum of the elements of $L$.

This method of constructing $K^{\prime}$ is similar to a method used by Zippin [8], but his result is not directly applicable here. 
separated sets $M_{1}$ and $M_{2}$ containing $p_{1}$ and $p_{2}$, respectively, and (2) every point of $K$ is a limit point of both $M_{1}$ and $M_{2}$ ?

THEOREM 2. If the decomposable compact meiric continuum $M$ is nearly 2-homogeneous and is not a triod, then $M$ is a simple closed curve.

Proof. It follows from Theorem 15 of [3] that $M$ is a continuous curve. Since $M$ is nearly homogeneous, it follows from Theorem 1 that $M$ is a simple closed curve.

CoROllary. If the nondegenerate compact metric continuum $M$ is 2-homogeneous and is not a triod, then $M$ is a simple closed curve.

Theorem 3. If the nondegenerate bounded continuum $M$ is homogeneously embedded in a plane $E$, then $M$ is a simple closed curve.

Proof. Suppose that $M$ is not a simple closed curve. It follows from two results by $F$. B. Jones $[4 ; 5]$ that some nondegenerate subcontinuum $K$ of $M$ is indecomposable. Mazurkiewicz [7] has shown that some point $y$ of $K$ is not accessible from the complement of $K$, and hence $y$ is not accessible from the complement of $M$. Since some point $x$ of $M$ is accessible from the complement of $M$, this leads to the contradiction that there is no homeomorphism of $E$ onto itself that carries $x$ into $y$ and $M$ onto itself.

\section{BIBLIOGRAPHY}

1. R. H. Bing, A homogeneous indecomposable plane continuum, Duke Math. J. vol. 15 (1948) pp. 729-742.

2. R. H. Bing and F. B. Jones, Another homogeneous plane continuum, Trans. Amer. Math. Soc. vol. 90 (1959) pp. 171-192.

3. C. E. Burgess, Continua and various types of homogeneity, Trans. Amer. Math. Soc. vol. 88 (1958) pp. 366-374.

4. F. B. Jones, Certain homogeneous unicoherent indecomposable continua, Proc. Amer. Math. Soc. vol. 2 (1951) pp. 855-859.

5. - On a certain type of homogeneous plane continuum, Proc. Amer. Math. Soc. vol. 6 (1955) pp. 735-740.

6. B. Knaster and C. Kuratowski, Problème 2, Fund. Math. vol. 1 (1920) p. 223.

7. Stefan Mazurkiewicz, Sur les points accessibles des continus indécomposables, Fund. Math. vol. 14 (1929) pp. 107-115.

8. Leo Zippin, $A$ study of continuous curves and their relation to the JaniszewskiMullikin theorem, Trans. Amer. Math. Soc. vol. 31 (1929) pp. 744-770.

UNIVERSITY OF UTAH 